\title{
Competencias y contenidos: cada uno en su sitio en la formación docente*
}

\author{
Competence and contents: each one in its own place in teacher training \\ Competências e conteúdos: cada um tem seu lugar na formação docente
}

\author{
Félix Angulo ${ }^{1}$, Silvia Redon ${ }^{2}$ \\ ${ }^{1}$ Universidad de Cádiz, felix.angulo@uca.es, móvil 34-686471954. \\ Departamento de Didáctica. Universidad de Cádiz. Puerto Real (España) \\ ${ }^{2}$ Pontificia Universidad Católica de Valparaíso, silvia.redon@ucv.cl, móvil 56-93787160, \\ Escuela de Pedagogía. Facultad de Filosofía y Educación. Viña del Mar (Chile) 2010
}

\begin{abstract}
RESUMEN
Este artículo tiene por objetivo analizar el concepto de "competencias" comenzando con las evidencias lingüísticas y pedagógicas de su confusión semántica, constatando ausencia de precisión y claridad, problemáticas que erróneamente han sido denominadas "complejas". En un segundo apartado se analiza el origen del término, vinculado al mundo de la empresa, políticas públicas educativas, a las que les subyace el mercado. Se discute la valoración de "novedoso" que envuelve al concepto de competencias, identificándolas como un recorte de otras teorías y contrastándolas con programas de UNESCO que demuestran que no hay muchos elementos de novedad en dicha propuesta. Por último, este artículo propone una reflexión curricular asentada en la ética y la phronesis, que nos lleva a preguntarnos: ¿Qué debemos enseñar? y por ende ¿Qué deben aprender las generaciones herederas del neoliberalismo, los estándares y las mediciones? ¿Desde dónde mirar los procesos formativos, subsumidos en la homogeneidad del mercado cultural-global?
\end{abstract}

Palabras clave: competencias, mercado, pseudo-complejidad y pseudoinnovación.

\begin{abstract}
This paper analyses the ideological, pedagogical and semantic confusion of the concept of competence, showing that it is not a complex concept but a concept under semantic confusion. In the second part we investigate the historical origins of the word, its connections with the ideology of the market and public policies that employ it. We emphasize that this is not an innovative concept, but it belongs to a long tradition of mechanistic approaches to human action. At the end we propose a reflection on the curriculum using the idea of phronesis, which leads us to ask ourselves the following questions: What should we teach? and therefore, What must the next generations inheritors learn about neoliberalism, standards and measures? From what point of view must we analyse the training processes, subsumed in the cultural-homogeneity of the global market?
\end{abstract}

Key words: competency, global market.

\section{RESUMO}

Analisa o conceito de "competências" a partir das evidências lingüísticas e pedagógicas da existência de uma confusão semântica relacionada ao termo, constatando a ausência de precisão e clareza nesse sentido - problemáticas que, inadequadamente, têm sido consideradas "complexas". Em um segundo momento, analisa a origem do termo, vinculando-o ao mundo empresarial, às políticas públicas educativas e à noção de mercado e o que aí está implícito. Discute-se a legitimidade da "nova" possibilidade de conceituar "competências", identificando-a como um recorte de outras teorias e contrastando-a com os programas da UNESCO que demonstram que não há muitos elementos novos em tal proposta. Por último, propõe uma reflexão curricular baseada na ética e na sabedoria prática aristotélica que nos leva a perguntar: $\mathrm{O}$ que devemos ensinar? Como devemos ensinar? O que precisam

* Este trabajo fue presentado al 1er Congreso Internacional. Reinventar la Profesión Docente. Universidad de Málaga. 2010. 
aprender as gerações herdeiras do neoliberalismo, a partir de quais padrões e em que medidas? De onde olhar os processos formativos, subjacentes à homogeneidade do mercado cultural global?

Palavras chave: competências, mercado, pseudo-complexidade e pseudo-inovação.

\section{LA CONFUSIÓN PRIMERO}

La presente comunicación responde a la perplejidad y sorpresa frente a la extensión y aceptación acrítica de la idea de competencias (Angulo 2009). Quizás lo que más nos llama la atención sea que el movimiento generado en los últimos años en pro de las competencias, recoja tanto a un sector importante de docentes e investigadores anteriormente partidarios de otras propuestas teóricas progresistas como el constructivismo, el aprendizaje situado y la pedagogía crítica, por poner algunos ejemplos, como a otros grupos mucho más conservadores, en una especie de conjunto o amalgama post-ideológica que parece pretender suspender el juicio y el análisis político (Bauman 2001, 2002).

La pregunta que se impone, más allá incluso de las condiciones líquidas (Bauman 2002) ${ }^{1}$ en las que vivimos, es la siguiente: ¿en qué se apoya epistemológicamente esta aceptación unilateral? Una parte de la respuesta se encuentra, a nuestro juicio, en la afirmación un tanto enfática, según la cual, competencia es un concepto complejo y por lo tanto multifacético y poliédrico (Pérez Gómez 2009). Sin embargo, se mire por donde se mire, ya sea en la versión que se ofrece para primaria y secundaria (teniendo como referente a $\mathrm{DeSeCo})^{2}$, o la que se ha extendido por la educación terciaria (con el Modelo Tuning como referencia), nos encontramos siempre con lo contrario: con un concepto confuso (De Ketele 2008) , $^{3}$, bajo cuyo paraguas semántico se engloba casi todo. Cuando formulamos la pregunta ¿qué queremos decir cuando hablamos de competencias? o ¿a qué nos referimos con ese término? (Angulo 2009), nos encontramos con un revoltijo conceptual. Perrenoud (2004: 11) ${ }^{4}$ afirma que competencia representa 'la capacidad de movilizar varios recursos cognitivos'; Hipkins (2006: 87) en el contexto de una propuesta curricular para Nueva Zelanda, afirma que "las competencias incluyen habilidades, conocimientos, actitudes y valores necesarios para presentar las demandas de la tarea"; Goñi Zabala (2007: 87), negando que el concepto deba abarcar habilidades, afirma a su vez que competencia es la capacidad 'para enfrentarse con éxito a una tarea en un contexto determinado'; y Blanco Blanco (2007) defendiendo una idea de competencia profesional sobre la que nos detendremos más tarde, afirma -al igual que Hipkins (2006)- que toda competencia "integra conocimientos, destrezas o habilidades y actitudes o valores" (2007: 36).

Abordar este panorama, nos obliga, como en tantas ocasiones en el mundo de la educación, a ir más allá de lo obvio y aparente. Está claro que afirmando que nos encontramos

\footnotetext{
$1 \quad$ Al referirnos a Bauman señalamos la liquidez como un cambio en la subjetividad de los individuos producto del impacto de la economía liberal en el mundo cultural, llámese trabajo, vínculos, redes sociales, itinerarios de vida, con ausencia de estabilidad, solidez, certezas y por tanto ausencia de autonomía y sujeto.

2 Definition and Selection of Competencies (DeSeCo).

3 De Ketele (2008) afirma que el concepto de competencia está mal definido, es poco claro y es epistemológicamente dudoso.

$4 \quad$ Uno de los participantes en la elaboración del documento DeSeCo, por cierto.
} 
frente a un concepto complejo (y no confuso) hacemos dos cosas: una, aceptar la confusión existente en el debate epistemológico y, por supuesto, político sobre el concepto y su utilización indiscriminada. Con ello, y en segundo lugar, la evitación del debate racional lleva, a nuestro juicio, la consecuencia más preocupante de dejar la puerta abierta para que la definición funcional del concepto quede en manos de instituciones, actuaciones y grupos internacionales de dudosa calidad y pertinencia educativa. ¿Qué queremos decir con lo de definición funcional? Con esta expresión queremos hacer referencia al hecho de que en última instancia la competencia será, nada más y nada menos, que aquello que marquen, determinen y, a fin y al cabo, definan dichas organizaciones e instituciones. Pongamos un ejemplo sencillo. Pérez Gómez (2007) es uno de los claros ejemplos que pretende realizar una lectura progresista de la idea de 'competencia', asociándola a una pedagogía activa y de fuerte impronta constructivista. En algún momento de su trabajo llega a afirmar que, en última instancia, la idea de competencia se asemeja a la de conocimiento práctico planteada por Schön (1983, 1987).

"el concepto de competencias aquí defendido, como habilidad para afrontar demandas externas o desarrollar actividades y proyectos de manera satisfactoria en contextos complejos, implica dimensiones cognitivas y no cognitivas: conocimientos, habilidades cognitivas, habilidades prácticas, actitudes, valores y emociones. Es un concepto muy similar al defendido por SCHÖN (1983, 1987) como característicos de los profesionales reflexivos: el conocimiento práctico" (Pérez Gómez 2007: 11) .

Incluso si aceptamos esta equiparación -que podría discutirse-, nada asegura que sea esta acepción de competencia la que se esté extendiendo y empleando. Por el contrario, basta revisar los trabajos de Goñi Zabala (2007), Hipkins (2006) y Blanco Blanco (2007), para ver que no es así ${ }^{6}$, y desde luego los documentos oficiales sobre los planes de estudio de primaria e infantil sobre los que volveremos más tarde.

La acepción funcional vigente se acerca a lo que Jones y Moore (1995-2008) denominan enfoque conductista o de observación directa de su desempeño (desagregación de las 'habilidades', la estandarización mesurable de resultados) en pro de un lenguaje de la transparencia ${ }^{7}$. DeSeCo (2005) -no lo olvidemos- es un programa de trabajo que ha llegado a constituir la base teórica y, a fortiori, conceptual, de una de las pruebas más extendidas en los sistemas educativos; nos referimos a las pruebas P.I.S.A.

"La evaluación PISA comenzó con la comparación del conocimiento y habilidades de los estudiantes en las áreas de matemáticas, ciencias y solución de problemas. La evaluación de la actuación del estudiante (performance) en materias escolares seleccionadas tuvo lugar con la comprensión de que el éxito en la vida del estudiante depende de un amplio rango de competencias. El Proyecto de Definición y Selección de Competencias de la OCDE, aporta un marco que puede guiar la extensión a largo plazo de la evolución hacia los dominios de la competencia" (DeSeCo 2005: 48).

\footnotetext{
5 Véase también Pérez Gómez (2009).

6 La situación no mejora en los textos canónicos de DeSeCo (2005) y Rychen y Hersh Salganik (2003) y Tuning (González y Wagenaar 2003, 2005) Véase Gimeno Sacristán (2009).

7 Un 'modelo de competencias' que se ajusta a unos objetivos económico políticos determinados. Dicho de otra manera, las competencias se inhabilitan como instrumento político, a medida que adoptan una forma más holística (Jones y Moore 1995-2008:6).
} 
Por ello, DeSeCo enfatiza que una competencia ha de ser una acción, una conducta o una elección particular manifiesta. Medir una competencia es medir inferencialmente a través de la constatación de su existencia en la actuación del sujeto. "La atribución de competencia es fundamentalmente una inferencia, realizada en razón de la evidencia que aporta la observación de una actuación" (DeSeCo 2005: 48).

No ha de extrañarnos, pues, que la que venimos denominando definición funcional recoja tanta adhesión. La evaluación -assessment- de los sistemas educativos, es un instrumento político de primer orden, una herramienta que está paulatinamente determinando las decisiones políticas de los países participantes (Angulo 1995). No hay que olvidar el liderazgo que ha asumido desde hace bastantes décadas en temas educativos -así como económicos- la OCDE (Organización para el Desarrollo Económico), como una organización que asume el control de las políticas educativas desde "mediciones" externas en los países involucrados (Gimeno 2008). Este afán por determinar y homogeneizar variables, indicadores, estándares o competencias nos conduce al currículum único, que instala en el imaginario social global una realidad incuestionable respecto de lo que debe ser "la calidad educativa" (Stake; 2006; Stobart 2010).

\section{TRAZOS DE UN NUEVO NEOCOLONIALISMO}

Si DeSeCo representa la macropropuesta para, fundamentalmente, la formación básica no universitaria, Tuning (Tuning Educational Structures in Europe) (González y Wagenaar 2003, 2006) es la propuesta para la educación terciaria (Angulo 2009, 2010). Éste no es un proyecto centrado exclusivamente en el diseño de competencias; en realidad, pretende algo más ambicioso: ofrecer a las universidades europeas esquemas eficientes de aplicación del 'programa de Bolonia'.

"El enfoque Tuning consiste en una metodología para rediseñar, desarrollar, implementar y evaluar programas de estudios para cada uno de los ciclos establecidos en Bolonia, de tal manera que pueda ser considerado válido mundialmente, puesto que ha sido testeado en varios continentes y probado su éxito" (ibid: 2006:1)

Tuning se crea para diseñar una nueva arquitectura que cumpla las líneas básicas establecidas en Bolonia y válida más allá de las fronteras europeas. Dicho en términos más técnicos: Tuning se ha propuesto ofrecer el modelo de diseño curricular esencial, estandarizado, válido y eficaz. A medio plazo, dicho modelo curricular estandarizado posibilitará comparar titulaciones y establecer criterios de acreditación y evaluación estándar.

Para ello, se requiere desarrollar perfiles profesionales para distintas enseñanzas universitarias a través de la especificación y selección de resultados de aprendizaje y "competencias deseables en términos de competencias genéricas y relativas a cada área de estudios incluyendo destrezas, conocimientos y contenido" (ibid: 2003: 31). El informe plantea una serie de justificaciones para el uso de competencias, y entre las más importantes (González y Wagenaar 2003: 34 y ss) se encuentran las siguientes:

1. Fomentar la transparencia en los perfiles profesional y académico de las titulaciones y programas de estudio, promoviendo un mayor énfasis en los resultados.

2. Desarrollo de un nuevo paradigma centrado en el estudiante y la necesidad de encauzarle hacia la gestión de conocimientos. 
3. Ampliar los niveles de empleabilidad y de ciudadanía.

4. Crear un lenguaje más adecuado para el intercambio y el diálogo entre los implicados.

El primero y el cuarto apuntan claramente hacia la operatividad de las burocracias estatales (Angulo 2010). La posesión de un lenguaje y una terminología común permite la comunicación fluida y genera a su vez que dicho diálogo se centre en los perfiles profesionales, transparentes $^{8}$ ahora porque sus características y elementos constitutivos son evidentes y los mismos, sea el país que sea en Europa y con independencia de la universidad europea de que se trate. Al igual que con la propuesta de DeSeCo, el modelo Tuning ha calado fuertemente en la conformación de los programas educativos universitarios, cumpliendo sobradamente estos puntos (el 1 y el 4).

Más dudoso se muestra el segundo, y todavía tendremos que esperar para ver si de verdad el modelo de competencias provoca en la universidad un cambio en la metodología docente y en los procesos de enseñanza aprendizaje, como algunos de sus apologistas han señalado (por ejemplo, Perrenoud 2004).

El tercer punto, el de la empleabilidad, se convierte en el punto medular: los perfiles profesionales se erigen en el nuevo artificio común para la educación superior en Europa en razón de que propiciarán, así se espera, la empleabilidad de los egresados y egresadas del sistema. No olvidemos que éste es, justamente, uno de los objetivos básicos de la Estrategia de Lisboa lanzada por los ministros y ministras de educación de los Estados Europeos en marzo de 2000, junto con su defensa de la economía del conocimiento. Dicha estrategia tiene por objetivo convertir a la Unión Europea en la economía más competitiva en el mundo, con la intención de alcanzar el pleno empleo en 2010 (European Council 2000) $)^{9}$. La estrategia descansa en tres pilares (Kok 2004):

- Un pilar económico orientado a preparar la transición europea a una economía basada en el conocimiento.

- Un pilar social, encargado de la modernización del modelo social europeo, invirtiendo en recursos humanos y combatiendo la exclusión social.

- Un pilar medioambiental, añadido en la reunión de Göteburgo de 2001, que intenta prestar atención a los recursos naturales y a un crecimiento sostenible (eco-industrias y eco-innovaciones).

El informe elaborado por Kok (2004) se muestra muy crítico con respecto al logro de los objetivos fijados. Pero, además de llamar la atención sobre la lentitud de su implantación, los remodifica añadiendo otros no menos importantes:

- Enfatizar el mercado interno, con la libre circulación de bienes y capitales, creando un único mercado de servicios.

- Reforzar el mercado laboral.

$8 \quad$ El deseo de transparencia es común a DeSeCo y a Tuning.

9 Un fracaso clamoroso a la luz de la situación que tanto nuestro país como otros países europeos está sufriendo, con altas tasas de desempleo y pérdida paulatina de apoyos sociales. Véase Judt (2010) y Navarro (2006). 
El tratado de Lisboa reconfigura el espacio de la teoría económica europea. Aunque se continúan declarando cuestiones relacionadas con la ciudadanía, la ecología y la democracia, lo cierto es que el terreno se ha desplazado de una política social o keynesiana a una política de intención neoliberal. Dar prioridad a la competitividad y, especialmente, a la economía basada en el conocimiento, no es más que adoptar formas encubiertas de neoliberalismo (Robertson 2008). Ello puede detectarse en, como decíamos, su defensa a ultranza de la economía del conocimiento.

Como ha indicado Godin (2006), el concepto de 'economía basada en el conocimiento' mantiene la retórica justificativa de que el conocimiento y las tecnologías de la información y la comunicación son factores clave para la economía; pero como Robertson (2008) señala, se trata de centrar los esfuerzos en un conocimiento que pueda generar beneficios económicos, por ejemplo, en forma de patentes. No nos equivoquemos, no se trata del conocimiento en un sentido amplio y comprensivo, se trata de que Europa se oriente hacia el conocimiento específico que genera utilidades y rentabilidades y no otro ${ }^{10}$.

"El imperativo de avanzar Europa como un proyecto político y económico y, en concreto, desarrollar una economía basada en el conocimiento, está unido al decline de Europa y de los Estados Unidos en la producción global de bienes. Por estas razones, tanto EE.UU. como UE comparten un interés común en la expansión de una economía global de servicios, incluyendo a la educación superior como un mercado, como una máquina para la innovación y un sector clave en el desarrollo de nuevas formas de propiedad intelectual" (Robertson 2008, p. 3).

¿Cómo realizar esta transformación? Porque, de alguna manera, las universidades europeas han mantenido un nivel importante de investigación, generación de conocimiento y relación empresarial e industrial. La Estrategia de Lisboa quiere acelerar y amplificar todo ello, pero no basta con afirmarlo y con extender las estructuras normativas aprobadas para las universidades. En este puzzle, necesitamos una tercera pieza, que Robertson (2008) indica pero que no desarrolla con el sentido en el que quiero darle aquí. La pieza es Tuning $(2003)^{11}$.

Si tenemos por un lado, un nuevo Espacio Europeo de la Educación Superior de la mano del Programa Bolonia y, por otro, un objetivo tan claro como el propuesto es la Estrategia de Lisboa sobre la economía del conocimiento, lo que se necesita en un traductor del conocimiento que se desarrolle dentro de las universidades en conocimiento, económica y mercantilmente viable (de Sousa Santos 2005). Tuning es ese traductor, porque entra en los contenidos de los planes de estudios universitarios y los modifica según perfiles profesionales, de la selección de resultados de aprendizajes y "competencias deseables en términos de competencias genéricas" (González y Wagenaar 2003, pág. 31) ${ }^{12}$. La introducción de este traductor significa mucho más que la conversión 'vocacionalista'

10 El informe Kok (2004) lo indica bajo el epígrafe de investigación y desarrollo de TICs (pág. 20).

11 Es muy probable que la otra gran pieza sea la European Qualifications Framework, lanzada en 2005 y sus homólogas nacionales. No podemos desarrollar este punto aquí, pero merece un análisis posterior. Nóvoa y de Johng-Lambert (2003), así lo entienden: "una diversidad de estrategias -benchmarking (comparación de rendimiento), establecimiento de objetivos, revisiones entre pares, redes de expertos, indicadores de rendimiento, etc.- son movilizados en orden a distraer la discusión de las cuestiones políticas y reorientarlas hacia los niveles más difusos de gobierno. El "estado regulador" implica al estado evaluador como tal, cuando los elementos de la economía de mercado son llevados al sector público” (pág. 46).

12 Angulo (2008), desarrolló las implicaciones de Tuning con mayor detalle. 
de la universidad, lo cual es evidente (Barnett 2002). En este punto tenemos que pensar que la cuestión es mucho más densa ideológica y políticamente que la búsqueda de la convergencia europea, transformar el lenguaje de las burocracias o fomentar la transparencia. La pretensión es provocar un giro neoliberal en la educación superior y someterla a las necesidades del mercado.

"Bajo la rúbrica de la economía basada en el conocimiento, la educación superior (en Europa) se valora ahora por su contribución económica y no cultural, como un mercado lucrativo y como un medio de generar nuevos valores a través de las patentes y la innovación" (Robertson 2006) ${ }^{13}$.

De Ketele (2000-2008), lo ha señalado también a su manera, cuando nos recuerda que no es una casualidad que "las primeras obras destacables en francés sobre las competencias han sido escritas por expertos que trabajaban en el mundo de la empresa, como Le Boterf (1994, 1997, 1998) y Lêvy-Leboyer (1996). Los expertos en educación se basaron ampliamente en sus obras (como Romainville, 1996; Perrenoud, 1997)".

Pero la estrategia así concebida apunta más allá del marco europeo. El modelo de competencia, a través del programa Tuning, se quiere erigir en, por así decir, un traductor universal exportable en sí mismo.

"El enfoque Tuning consiste en una metodología para re-diseñar, desarrollar, implementar y evaluar programas de estudios para cada uno de los ciclos establecidos en Bolonia, de tal manera que pueda ser considerado válido mundialmente, puesto que ha sido testado en varios continentes y probado con éxito" (González y Wagenaar 2006, pág. 1).

Por ello, existe un Tuning Latinoamericano, Ruso y Asiático. El modelo de competencias, tal como Tuning lo plantea, se extiende como si se tratase de un nuevo y refundado colonialismo, un modelo universal y en tanto tal, rentable, de organización empresarial universitaria.

"Mientras por mucho tiempo Europa ha legitimado sus actividades presentándose a sí misma como una presencia más civilizadora que imperialista... Sus intereses más explícitos: económicos y transnacionales, la sitúan en el plano de un nuevo colonialismo e imperialismo". (Robertson 2006: 39) ${ }^{14}$.

La literatura al respecto es tan copiosa como inabarcable ${ }^{15}$, y para no extendernos quisiéramos citar únicamente el ejemplo del caso chileno. En Chile como en muchos de los países latinoamericanos existe una gran demanda por diseñar los currícula de formación profesional basados en competencias ${ }^{16}$. Presionados por estas políticas públicas, se han generado los recursos, plataformas y fondos ministeriales para satisfacer dichas demandas. Cabe destacar que en estas políticas nacionales, que involucran grandes sumas de dinero, están detrás bajo el velo de la cooperación y asistencia técnica organismos transnacionales como OCDE; BM; FMI; IESALC (Fonseca 1995, cit. en Gentili, 1996: 14).

13 No olvidemos que la economía neoliberal, a través de la educación, transforma la 'subjetividad' del individuo en algo vacío de sí mismo, ahistórico, carente de sentido por lo común, y por la capacidad por repensar, recrear y transformar el orden impuesto por la econometría mundial (Véanse Beck; Beck-Gernsheim 2003, Bauman 2002, Sennett 2006; Castoriadis 2002; entre otros).

14 Véase también Hartmann (2008).

15 Véase Cepeda Dovala (2010) y Daniele Paranhos y Rino Mendes (2010).

16 Especialmente los países y universidades reunidos por el proyecto ALFA Tuning Latin America Project. (http://tuning.unideusto.org/tuningal/index.php?option=content\&task=view\&id=168\&Itemid=196) Visitado 21 de septiembre de 2010. 
El mecanismo "oficial" por el cual universidades chilenas acceden a proyectos vinculados con la renovación y mejora de sus currícula, se denomina MECESUP (Mejoramiento de la Calidad de la Educación Superior) Aunque estos fondos se reparten entre diversos objetivos (contratación de profesionales doctores y actualización bibliográfica) una parte considerable de los mismos se destina al asesoramiento para la formulación de competencias en los planes de formación docente y en la elaboración de los currícula universitarios. En 2004 se destinaron a tal efecto unos nueve millones de dólares (un 41\% del presupuesto general) y entre los años 2006 y 2008 el monto total ascendió a 13 millones y medio de dólares, i.e., más de un 50\% del presupuesto global para esos años ${ }^{17}$.

Sin dejar de valorar la inversión pública que hace el Estado chileno para la mejora de sus currícula de formación profesional, es lamentable que una corriente atrapada políticamente en una ideología neoliberal, además de controvertida, poco clara, confusa y débil epistemológicamente (de Ketele, 2000), se convierta en el sello de "calidad" de la formación de los y las profesionales chilenos/as, exacerbado por el abultado gasto que ha implicado.

Este capital económico global presente en los mercados transnacionales como ejes de poder políticos por sobre los Estados nación (FMI, BM, OCDE), requiere del monopolio de la ciencia, la tecnología y la cultura para su hegemonía. Por ello ha conquistado a las universidades como nicho del colonialismo del saber a través de la homogenización de sus planes de formación profesional en el marco Tuning y los currícula por competencias: "Se presentan algunas evidencias que (de)muestra el trasfondo ideológico que motiva el trasplante e imposición del enfoque llamado de 'competencias' desde el canon de la lógica del capital hacia el campo educacional. Tal discurso del poder hegemónico neoliberal tiene un origen y filiación economicista y está impregnado de significación pragmática y utilitaria. La reducción del quehacer educativo a su mera mercantilización e instrumentación tecnocrática y conductista atenta contra una concepción educativa humanista, integral, democrática e inclusiva, que postulamos como la pertinente para Chile y nuestra América". (Rubilar 2009) Estas políticas no son más que una ejemplo evidente de los procesos de neocolonialismo procedentes de Europa y Norteamérica; procesos que además de la 'ocupación' económica a manos de empresas multinacionales (como las telefónicas), o del tratado de libre comercio con Norteamérica, implican también la imposición de ideas y procedimientos culturales y educativos poco o nada sensibles con la historia, las necesidades y contextos latinoamericanos propios.

\section{EL CONOCIMIENTO CULTURAL Y SU PÉRDIDA}

Después del recorrido que hemos realizado hasta aquí, quisiéramos centrarnos en uno de los puntos que han estado latiendo en los dos apartados anteriores. Nos referimos a la desaparición o invisibilización, a nuestro juicio lamentable, del conocimiento cultural y aun académico en las nuevas concepciones sobre los planes de formación universitaria y, especialmente, en los de la formación de docentes. Para entender, repetimos, esta

17 Cifras públicamente difundidas por el Ministerio de Educación en su plataforma oficial. 
pérdida o invisibilización, tenemos que situarnos de nuevo tanto en las propuestas de DeSeCo como en la de Tuning, enfocando la mirada a sus intersticios más concretos. Comencemos por un cuadro que nos puede servir de comparación.

Ya hemos señalado que DeSeCo define su enfoque como funcional y orientado a las demandas. Son las demandas de la vida cotidiana que cada alumno o alumna tendrán que afrontar -demandas que se suponen variadas- las que determinan -aquí- la idea de competencia ${ }^{18}$. Para ello, DeSeCo selecciona tres grandes ámbitos que constituyen las competencias clave:

- Uso interactivo de herramientas

- Necesidad de interaccionar en y con grupos heterogéneos.

- Actuar autónomamente

El primer grupo hace referencia a herramientas tan diversas como el lenguaje y las nuevas tecnologías para poder interactuar con el ambiente. El segundo grupo apela al hecho de que en un mundo 'crecientemente interdependiente', los individuos tienen que relacionarse con otros grupos e individuos heterogéneos y diversos. El tercero se orienta a las 'responsabilidades que los individuos han de adquirir en sus propias vidas, situándolas en los contextos sociales y actuando con autonomía' (DeSeCo 2005: 5). Resulta imposible no estar más de acuerdo con estos planteamientos, sin más adjetivación. Sin embargo, en ellos no aparece la idea de contenido cultural, lo que debería resultar lógico viendo cómo se entiende el concepto de competencia.

No obstante, si escarbamos un poco más, podemos encontrar que estas ideas básicas proceden, esencialmente, de otra propuesta anterior recogida en el Informe Delors (UNESCO 1996) ${ }^{19}$. En él, se recordará, con la idea de estimular 'la creatividad' en la enseñanza, se distinguía cuatro ámbitos esenciales para la educación del siglo XXI:

- Aprender a conocer (i.e. desarrollo de la razón).

- Aprender a hacer (i.e. habilidades prácticas y técnicas).

- Aprender a vivir juntos (i.e. habilidades de comunicación).

- Aprender a ser (i.e. habilidades de gestión de la propia vida).

Según los autores de este informe, la educación debería trascender, por lo tanto, "la visión puramente instrumental de la educación considerada como la vía necesaria para obtener resultados (dinero, carreras, etc.)", reconsiderando la función que tiene en su globalidad y el papel que juega en la 'realización de la persona, que toda entera debe aprender a ser' (UNESCO 1996: 76). Y es justamente esa visión puramente instrumental, la que la propuesta DeSeCo abraza y conjuga.

18 El aspecto práctico que subyace a la idea de competencia manejada por DeSeCo es hipertrofiado y aumentado, probablemente de modo intencional, en la definición que de competencia se utiliza en Pérez Gómez (2007: 10).

19 Puestos a buscar 'arcanos' podemos cotejar esta ideas con el, ya olvidado, Informe de la Comisión Fauré de 1972, también de la UNESCO. 
Detengámonos un momento en el cuadro siguiente:

\section{Cuadro 1}

\begin{tabular}{|l|l|}
\hline Delors (1996) & DeSeCo (2003) \\
\hline Aprendizajes genéricos & Competencias clave \\
\hline Aprender a conocer & Uso interactivo de herramientas \\
\hline Aprender a hacer & \\
\hline Aprender a vivir juntos & Interactuar en grupos heterogéneos \\
\hline Aprender a ser & Actuar autónomamente \\
\hline
\end{tabular}

Como puede verse, en DeSeCo desaparece aprender a conocer que está en Delors, mientras que los restantes son prácticamente similares. Supongamos por un momento que la idea de DeSeCo se encuentra en simplificar la propuesta, aceptando -epistemológicamente- que conocer, es decir, desarrollar la razón y adquirir conocimientos culturales para comprender el mundo es lo mismo que usar herramientas interactivamente ${ }^{20}$. Sin embargo, también desde un enfoque meramente epistemológico, adquirir el lenguaje y habilidades, o competencias informáticas, que es de lo que se trata cuando emplean el concepto de herramienta, no puede ser sinónimo de adquisición de una sólida estructura conceptual (teorías, modelos, enfoques, etc.) sobre el mundo (Young 2008 a , 2008b; Donald 2002; Tomasello 2007).

Quizás debamos recordar lo que señala Donald (2002: 35): "La relación entre cultura y conciencia es recíproca. Mientras la cultura emerge de los intentos de una conciencia expandida por conectar con otras, es la inmersión en la cultura, mucho más que cualquier factor del cerebro, lo que define nuestra auténtica conciencia humana".

Ahora podemos entender ciertas cuestiones que hacen del concepto de competencia un concepto altamente confuso y dado a atropelladas definiciones. Por ejemplo, cuando equipara competencia con conocer; algo que raya en el absurdo epistemológico. No creo que sea necesario volver a repetir lo que acabamos de mencionar: poseer herramientas, por ejemplo, cognitivas no obvia el adquirir estructuras de conocimiento cultural. Sin embargo, en los planes de estudio vigentes en las dos diplomaturas aprobadas: primaria e infantil, este tipo de errores se repite una y otra vez.

En España, la Orden ECI/3857/2007 para la verificación del título de Maestro en Educación Primaria, afirma en su Apartado 3, denominado Objetivos, lo siguiente: “competencias que los alumnos estudiantes deben adquirir", con lo que el documento legal equipara objetivos y competencias. O dicho de otra manera, ya no hay objetivos como tales, sino sólo competencias, aunque, claro, se sigue empleando el primer concepto como genérico. Lo que nos podría llevar a la conclusión contraria: las competencias

20 La falta de novedad de la propuesta de DeSeCo es también señalada por Col y Martin (2006). Visiones alternativas y espitemológicamente mucho más sólidas, pueden encontrarse en Donald (2002), Cole (2003), Engeström (1987) y Engeström, Miettinen \& Punamäki (1999). 
son objetivos. Si así fuera, quizás la confusión sería menor. Queremos decir que el concepto de objetivo es independiente con respecto a sus propios contenidos. Objetivo trata de indicar una meta, un resultado, un punto final que se ha de alcanzar. En este sentido puede ser tanto un contenido, como una conducta, habilidad u otra cosa que queramos indicar; recuérdense las taxonomías de objetivos de Bloom. Ya sabemos, también, que los objetivos tienen su propia historia y servidumbre (Gimeno Sacristán 1982), pero, repetimos, tenían la virtud al menos de mostrarse independientes ${ }^{21}$ con respecto a lo que quisiéramos denominar objetivo. El problema que presentaban era su propia definición como objetivos terminales, operativos y conductuales y el modelo medios/fines i.e. técnico que representa para el diseño y desarrollo del curriculum (Gimeno 1982; Angulo 1994).

La cuestión es otra con competencia; porque por mucho que queramos ampliar el campo de lo que puede ser o es competencia, para que todo quepa en ella, en tanto concepto la competencia apunta a un tipo de acción, o si se quiere está dirigida a la actuación, al dominio de habilidades, a la performance, como son las que señala DeSeCo (véase cuadro $n^{\circ} 1$ ). En ningún caso conocer algo puede ser considerado como competencia, a menos que aceptemos que poseer estructuras de conocimiento es en sí mismo una competencia, lo que no deja de resultar una contradictio in terminis. Lo mismo podríamos decir de otras 'competencias' que se indican en el documento oficial, como por ejemplo las siguientes, de las que citamos los encabezados:

- Conocer áreas curriculares.

- Diseñar, planificar y evaluar procesos de enseñanza y aprendizaje

- Abordar con eficacia situaciones de aprendizaje en contextos multiculturales.

- Diseñar y regular espacios de aprendizaje en contextos de diversidad.

- Fomentar la convivencia en el aula y fuera de ella.

- Conocer la organización de los colegios de educación primaria y la diversidad de acciones que comprende su funcionamiento.

- Mantener una relación crítica y autónoma respecto a los saberes, los valores y las instituciones sociales, públicas y privadas.

Tendríamos que preguntarnos si conocer las áreas curriculares es realmente una competencia, lo mismo pasa con 'conocer la organización de los colegios'. Ambos pueden ser objetivos de la formación primaria que implican la adquisición de estructuras de conocimiento disciplinar. Pero ¿qué decir de diseñar, planificar y evaluar? En este objetivo se entrecruzan tanto conocimiento disciplinar como habilidades, experiencias, conocimiento tácito (o práctico, si se quiere) y competencias de actuación. Pero desde luego, sin un conocimiento de distintos modos, modelos y 'experiencias' de diseño, planificación y evaluación, nos resulta poco creíble desarrollar cualquier tipo de competencia. Los objetivos de 'abordar con eficacia' y 'fomentar la convivencia' presentan sus propias dificultades como competencia. Con independencia de que habría que definir qué significa eficacia en un contexto práctico de enseñanza, tendríamos aquí también que

21 Está claro que no cualquier cosa podría ser un objetivo. El requisito era externo: sólo podría ser un objetivo de la educación aquello sobre lo que pudiese constatarse su adquisición. (Gimeno ) 
generar procesos analíticos (además de experienciales) y reflexivos en los que se ha de conjugar conocimiento disciplinar; lo que vale para el objetivo de la convivencia: fomentarla también exige conjugar una extraordinaria experiencia práctica, análisis y reflexión donde intervienen habilidades, conocimientos disciplinares, morales y experienciales. Por último, el objetivo de mantener una relación crítica y autónoma tiene tanta fuerza ético moral que no puede, más que forzando y tergiversando su sentido, convertirlo en competencia.

Desde luego, repetimos, si éste fuera el problema básico con el empleo de las competencias en la formación docente, no supondría un problema tan grave; al fin y al cabo representaría otro más de los dislates a los que nos tiene acostumbrados el legislador $^{22}$. Lo preocupante es que estos objetivos que erróneamente denominan competencias han dado lugar a unas 69 subcompetencias en las que se desglosan (para su inclusión en las asignaturas), elaboradas fundamentalmente por la $\mathrm{ANECA}^{23}$. La confusión se convierte ahora en un disparate. ¿Cómo, seriamente, podemos denominar competencia a "analizar y comprender los procesos educativos en el aula y fuera de ella relativos al período 6-12", a "conocer las propuestas y desarrollos actuales basado en el aprendizaje de competencias" o a "conocer la evolución histórica del sistema educativo en nuestro país y los condicionantes políticos y legislativos de la actividad educativa", y así sucesivamente hasta, como decimos, 69 subcompetencias? ¿Cómo podemos aceptar que con la adquisición de dichas 69 subcompetencias (transferibles y homogéneas para la titulación) tendremos docentes formados? ¿Cómo podemos creernos que podremos formarlos con ellas? 24

Si tomamos Tuning como referencia, la situación no mejora en absoluto. Comencemos por decir que, a pesar de que ha sido y es Tuning el elemento clave de la transformación de los planes de estudios en estructuras de competencia, como tal no aparecen en los documentos oficiales. Parece que sirvieran más como justificación científica (algo que resulta poco convincente dada su débil base epistemológica) (Angulo 2009) y política, que como modelo concreto de toma de decisiones. Veamos las competencias genéricas que Tuning indica ${ }^{25}$.

22 Parece que hemos olvidado que la ley española L.O.G.S.E (Ley Orgánica General del Sistema Educativo) definía el concepto de currículum, y que en los contenidos se distinguía entre hechos, conceptos, procedimientos y actitudes, con la intención de ser incluidos en las programaciones y los diseños curriculares. Véase Col et alli (1992).

23 ANECA es acrónimo de la Agencia Nacional de Evaluación de la Calidad y Acreditación. La agencia ha publicado un libro blanco por cada titulación. Pueden consultarse en: http://www.aneca.es/publicaciones/ libros-blancos.aspx

24 Una pregunta muy parecida a las que formulaban los críticos cognitivos de la formulación operativa de objetivos. Véase Gimeno Sacristán (1982).

25 Compárense estas 'competencias' con las que aparecen en el Informe Final Proyecto Tuning-América Latina 2004-2007. 
Cuadro 2

Competencias Genéricas

Tuning Educational Structures in Europe ${ }^{26}$

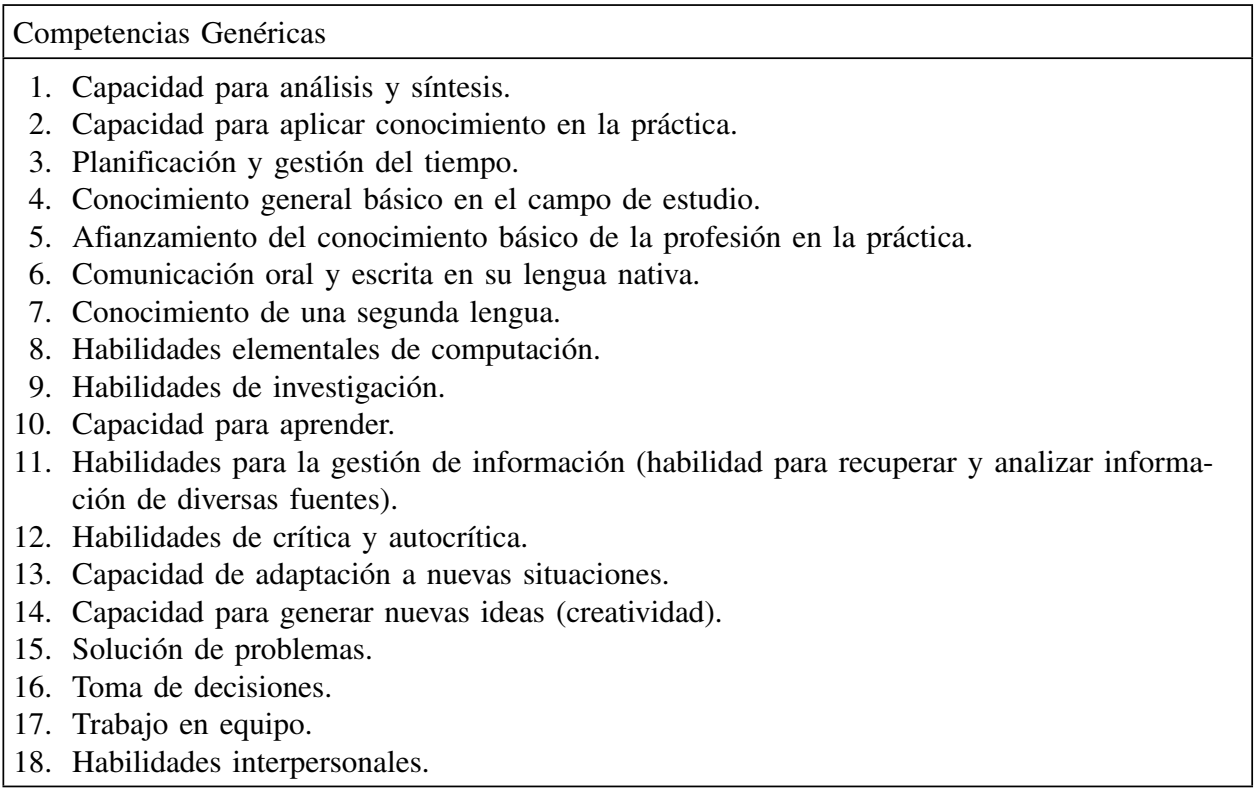

Lo que parece claro, después de una lectura detenida de la tabla es que sólo como recurso retórico se puede decir que los 18 componentes del cuadro $\mathrm{n}^{\mathrm{o}} 2$ son competen$\operatorname{cias}^{27}$. Se habla de capacidades, de habilidades, de solución de problemas, que-volvemos a repetir- sólo forzando el concepto de competencia se puede aceptar su equiparación. Pero conocer es conocer, i.e. dominar y poseer estructuras disciplinares de conocimiento que nos servirán de fundamento para dialogar con el mundo a nuestro alrededor y comprenderlo. Lo mismo pasa con la creatividad; la creatividad, ser creativo, no es una competencia, ni depende exclusivamente del dominio de habilidades, técnicas o procedimientos (Egan 1994). La creatividad es algo mucho más complejo que la competencia y no es tan fácilmente evaluable, como -según sus defensores- debería serlo.

De todas maneras a nuestro juicio, y por mucho que continuemos analizando el entramado retórico de las propuestas en las que ha tomado cuerpo el movimiento de

26 Los componentes de este cuadro fueron tomados originalmente en noviembre de 2009. Actualmente la página web del proyecto Tuning presenta 30 competencias. Entre las nuevas destacan: Habilidad para trabajar autónomamente, Espíritu de iniciativa y de emprendimiento, compromiso ético y Voluntad de éxito. Afirmar que el trabajo autónomo, la iniciativa, el compromiso y la voluntad, son competencias representa uno de los mayores dislates epistemológicos que Tuning ha mostrado a lo largo de su desarrollo.

27 En el documento para Latinoamérica las competencias genéricas se amplían a 27. Entre las que están la capacidad de motivar y el compromiso con el medio ambiente. Nos encontramos con los mismos problemas que hemos mostrado antes. Una capacidad y un compromiso no son una competencia. 
competencias, no entraremos en su problema medular, en el centro del peligro que representan: la pérdida y enmascaramiento de conocimiento cultural y disciplinar. Como hemos visto en los ejemplos anteriores, ya no se habla de disciplinas del conocimiento: no existe la antropología, la sociología, ni la psicología, ni, desde luego, la psicología social, la psicología del aprendizaje, o la sociología de la educación ${ }^{28}$. Ya no digamos la Historia de la Educación, la Didáctica y la Organización Escolar, o la Metodología de Investigación. Sólo existen competencias: 69 en total.

Este enmascaramiento, que sentimos como pérdida, ha sido denunciado en la formación vocacional o profesional justamente en relación a la formación basada en competencias, extendida en ese campo educativo desde los años 80 (Wheelahan (2007) ${ }^{29}$.

"El acceso al conocimiento disciplinar es importante por razones epistémicas, porque aporta a los y las estudiantes el acceso a las 'representaciones colectivas' sobre los mecanismos causales de las disciplinas estudiadas, mecanismos que no siempre son accesibles a través de la experiencia directa (o la solución de problemas). Las disciplinas aportan a los estudiantes acceso a la conexiones relacionales en un campo de estudio y entre campos, y los estudiantes necesitan acceder a 'estilos de razonamiento' disciplinar... Si el mundo está caracterizado por la profundidad ontológica, la estratificación, la emergencia y la codeterminación, los estudiantes necesitan conocer dichos procesos, y no poseer dichas comprensiones restringe su acceso a los acontecimientos y a las experiencias" (Wheelahan 2007: 640).

Argumentos parecidos ha planteado recientemente Young (2008a), quien señala con claridad: "la adquisición de conocimiento es la clave que distingue a la educación, tanto sea básica, profesional o universitaria de otras actividades", y añade: un "curriculum para el futuro' necesita tratar el conocimiento como un elemento distintivo y no reducible a los recursos cambiantes que la gente necesita para acceder y a dar sentido al Mundo" (Young 2008b: 80).

Incluso César Col y Elena Martín (2006) ${ }^{30}$, en un trabajo reciente que lleva el significativo título de Vigencia del debate curricular, afirman lo siguiente:

"La identificación y definición de los aprendizajes esperados de los alumnos en términos de competencias no permite prescindir de los contenidos. Es más. Definir los aprendizajes básicos únicamente en términos de competencias puede ser engañoso, ya que su adquisición va siempre asociada a una serie de saberes (conocimientos, habilidades, valores actitudes), que, no por el hecho de estar implícitos, dejan de estar implicados" (18).

Las competencias no pueden pues prescindir de los contenidos culturales, los saberes o los contenidos disciplinares (Torres 2008). Cuando los invisibilizamos, imponiendo el modelo de competencias vigente, estamos eliminando el debate epistemológico y político sobre qué queremos como sociedad que nuestras futuras generaciones aprendan y qué conocimientos han de adquirir los futuros profesionales. Es más, anulando el papel de

28 Esta cuestión se refleja de forma evidente en los así denominados Módulos de los títulos universitarios de Primaria e Infantil. Véase Orden ECI/3857/2007.

29 Wheelahan (2007) señala que el lenguaje progresista en educación ha estado asociado con la Competencybased teaching en Australia (640).

30 Col y Martín (2006) distinguen con cierta claridad entre saberes básicos y competencias clave. Sin embargo, confunden aprendizajes con saberes. 
los contenidos disciplinares en la formación docente estamos contribuyendo a su degradación profesional.

"La introducción de la competencia desprofesionaliza las clases y reemplaza las formas democráticas y colegiadas en la organización y en el control del trabajo por otras técnicas y gerencialistas. El sector del "mundo del trabajo" más afectado por las competencias es aquel formado por los propios profesores y formadores" (Jones \& Moore 2008: 14).

Las competencias sitúan a los docentes en un plano técnico, es decir, los descualifican profesionalmente; al traducir en forma transparente -medibles, cuantificables y acumulables- las habilidades profesionales, las estamos exponiendo al control de los gestores, a su mercantilización y a la descualificación neoliberal de la profesión docente; una, como decía, vuelta política al más burdo taylorismo.

Dichos profesionales no requerirán conocimiento disciplinar de ningún tipo -da a entender esta postura- para el ejercicio de su profesión; les basta con la adquisición de una serie de competencias (69 para ser exactos). Por ello, habría que dejar en manos de otros profesionales el dominio de dichas estructuras. De esta manera, no lo olvidemos, podemos derivar a situaciones en las que para ejercer la docencia, así como para formar a docentes, bastará con acreditar el dominio de dichas competencias y, actualmente, sólo agencias externas sin control democrático y público alguno son las encargadas de dicho proceso de acreditación. A la larga, las competencias nos convertirán, si no lo somos ya, en una sociedad auditada (Power 1999).

Las consecuencias de este proceso de transformación líquida (Bauman 2002) -o fisión, para emplear la expresión física correcta- pueden ser todavía mucho más graves si nos detenemos en los países latinoamericanos, como sugeríamos más arriba. La exclusión o supresión del debate sobre los contenidos culturales en los currícula nacionales, tiene aquí un impacto enorme. Los países colonizados, como el caso del conglomerado latinoamericano, han recorrido un camino difícil y complejo en la constitución de sus Estados nación. Poseen un tejido social fragmentado, producto de un itinerario histórico con profundas carencias de memoria colectiva e invisibilización de las culturas originarias, conviviendo con la cultura dominante impuesta por las clases oligárquicas en la mayoría de los países latinoamericanos. El proceso de expansión del mercado global ha utilizado esta realidad latinoamericana incorporando a la educación en estos procesos de mercantilización para perpetuar la hegemonía cultural y, entre ellos, especialmente el movimiento de competencias.

\section{VOLVER A LA PHRÓNESIS}

No quisiéramos terminar este trabajo sin dar un paso más. Hasta ahora hemos ido analizando el sentido y la aplicación de la idea de competencia en general y, específicamente, en la formación docente. Hemos indicado con insistencia que una de las pérdidas más preocupantes se encuentra en el enmascaramiento del debate epistemológico y político sobre los contenidos, tanto en la educación básica como en la formación de docentes (Torres 2009). Con la misma fuerza con la que hemos defendido la importancia de los contenidos, queremos afirmar que de la misma manera que con la adquisición de competencias no formamos docentes, con la simple adquisición de conocimientos disciplinares y saberes -si se prefiere- tampoco. Hipertrofiar sin más el papel de los 
conocimientos lleva el peligro asociado de derivar en lo que, no sin razón, se ha criticado como academicismo; i.e. una formación alejada de las complejidades de la práctica (Angulo 1993). A su manera, el academicismo es otra forma de tecnicismo, porque conlleva la idea -implícita- de que con la adquisición de teorías y modelos, podremos resolver los retos de la práctica docente. Por su lado, sobreestimar las competencias nos lleva al otro extremo: hacernos creer que con la adquisición de las mismas -en forma de habilidades, rutinas, solución de problemas, etc.-, estaremos formados y preparados para hacer frente a una práctica educativa enormemente compleja, que es convertir la práctica profesional en mera cuestión técnica.

La manera de transitar entre la Escila academicista y el Caribdis técnico, ha sido ya formulada y pertenece a lo mejor de la tradición pedagógica y de formación docente, con autores como Stenhouse (1978), Schön (1983, 1987), Elliott (1990), Grundy (1987), que nos ayudaron en otros tiempos a comprender que la mejor formación docente se encuentra en el desarrollo del conocimiento práctico o del juicio profesional práctico ${ }^{31}$. Actualmente Toulmin (2003) y Flyvbjerg (2001) desde el campo de la epistemología y Dunne (2001) de la filosofía de la educación, nos lo han vuelto a recordar a través de su defensa de la idea de phronesis. Para explicar, aunque sea brevemente, esta idea, veamos la imagen siguiente.

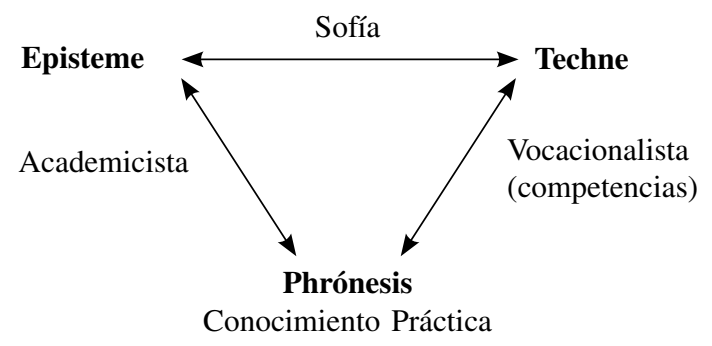

Aceptemos que episteme representa al conjunto de conocimientos disciplinares y techné a las habilidades, rutinas, técnicas concretas que han de adquirir los futuros docentes (Toulmin 2003). En la formación no se puede prescindir de ninguna de ellas, como hemos dicho; pero hemos de conjugarlas de tal manera que nutran y eduquen la phrónesis del alumnado y del futuro docente. La phrónesis o prudentia, representa ese punto en el que tocamos los dilemas y exigencias y complejidades de la práctica docente. Afrontamos la práctica desde estructuras de conocimiento disciplinar y grupos de habilidades que poseemos y dominamos, y son ambas desde y con las que conversamos y dialogamos con la práctica en las exigencias de la acción (Schön 1983, 1987). La reflexión sobre ese diálogo y conversación educa nuestra phrónesis, reformula nuestro conocimiento y nos provee con nuevas habilidades técnicas en un proceso constante; i.e. desarrolla nuestro conocimiento práctico. La formación universitaria de los futuros docentes tiene la obligación de desarrollar su conocimiento práctico, su phrónesis. La sola apelación al

31 Angulo (1999) es una revisión in extenso de esta corriente. 
conocimiento académico no lo logrará; mucho menos la insistencia en las competencias. Así pues, regresemos a la phrónesis, discutamos su sentido y pongamos al contenido y a las competencias cada uno en su sitio.

\section{REFERENCIAS BIBLIOGRÁFICAS}

Angulo Rasco, José Félix (1993). ¿Qué profesorado queremos formar? Cuadernos de Pedagogía. $\mathrm{n}^{\mathrm{o}}$ 220: 36-39.

Angulo, José Félix (1994). Enfoque tecnológico del curriculum, en Angulo, J. Félix y Blanco, Nieves (1994) (Comp.) Teoría y desarrollo del curriclum. Málaga. Aljibe: 79-110.

Angulo, José Félix (1995). La evaluación del sistema educativo: algunas respuestas críticas al porqué y al cómo, en AA.VV. (1995) Volver a pensar la educación. (Vol. 2) Prácticas y discursos educativos. Madrid. Morata: 194-219.

Angulo, José Félix (1999). De la investigación sobre la enseñanza al conocimiento docente, en Angulo, J.F. Barquín, J. y Pérez Gómez, A. Ignacio (Comp.) Desarrollo profesional del docente. Política, investigación y práctica. Madrid. Akal: 261-319.

Angulo, José Félix (2009). La voluntad de distracción: las competencias en la universidad, en Gimeno Sacristán, José (Comp.) (2008). Educar por competencias, ¿qué hay de nuevo? Madrid. Morata: 176-205.

Angulo, José Félix (2010). La educación y el curriculum en el espacio europeo: ¿internacionalizar o globalizar?, en Gimeno Sacristán, José (Comp) (2010). Saberes e incertidumbres sobre el curriculum. Madrid. Morata: 478-498.

Bauman, Zygmunt (2001). La postmodernidad y sus descontentos. Madrid. Akal.

Bauman, Zygmunt (2002). Modernidad líquida. Buenos Aires. Fondo de Cultura Económica.

Bauman, Zygmunt y Tester Keith (2002). La ambivalencia de la Modernidad y otras conversaciones. Paidós. Barcelona España.

Castoriadis, Cornelius (2002). La insignificancia y la imaginación. Trotta. Madrid.

Cepeda Dovala, Jesús Martín (2010). Metodología de la enseñanza basada en competencias. Universidad Autónoma del Noroeste. México. Revista Iberoamericana de Educación (ISSN: 1681-5653).

Col, César et alli (1992). Los contenidos en la reforma. Enseñanza y aprendizaje de conceptos, procedimientos y actitudes. Barcelona. Santillana.

Col, César y Martín, Elena (2006). Vigencia del debate curricular. Aprendizajes básicos, competencias y estándares. II reunión del Comité Intergubernamental del Proyecto Regional de Educación para América Latina y el Caribe (PRELAC). Santiago de Chile. 11-13 de Mayo de 2006. Documento no publicado. URL: http:!!www.ub.edu/gintrie (21/09/10).

Cole, Michael (2003). Psicología Cultural. Madrid. Morata.

Daniele Paranhos, Vania y Rino Mendes, María Manuela (2010). Currículo por competencia y metodología activa: percepción de estudiantes de enfermería, Revista Latino-Americana Enfermagem. 18 (1). http://www.eerp.usp.br/rlae (visitado 10 de Septiembre de 2010).

De Ketele, Jean Marie (2000). "Approche socio-historique de competences dans l'enseignement", en Quel avenir pour les competences? Bruxelles: DeBoeck 2000.

DeSeCo (2005). The definition and selection of key competencies. Executive summary. http:// deseco.admin.ch (consultado 27/05/05).

De Sousa Santos, Boaventura (2005). La universidad en el siglo XXI. Para una reforma democrática y emancipadora de la universidad. Buenos Aires. Miño y Dávila.

Donald, Merril (2002). A Mind so rare. The Evolution of Human Consciousness. New York. W. W. Norton \& Company. 
Dunne, Joseph (2001). Back to the rough ground. Practical judgement and the Lure of technique. Notre Dame, Indiana. University of Notre Dame Press.

Egan, Kirian (1994). Fantasía e imaginación: su poder en la enseñanza. Madrid. Morata.

Elliott, John (1990). La investigación-acción en educación. Madrid. Morata.

Engeström, Y. (1987). Learning by expanding: An activity-theoretical approach to developmental research. Helsinki: Orienta-Konsultit.

Engeström, Y., Miettinen, Reijo \& Punamäki, Raija-Leena (1999). Perspectives on Activity Theory. Cambridge. Cambridge University Press. (1987).

Fonseca, M.O. (1995). Banco Mundial e a educaçao: reflexoes sobre o cáso brasileiro. en: Sader, E. \& Gentili, P, (ed) (1995). Pós-Neoliheralisino As politices sociais e o estado democrático. Paz e Tena, São Paulo.

Flyvbjerf, Bent (2001). Making Social Science Matter. Why social inquiry mails and how it can succeed again. Cambridge. Cambridge University Press.

Gentili, P. (1996). El Consenso de Washington y la crisis de la educación en América Latina. Paper. Universidad del Estado de Río de Janeiro. Brasil. http://www.ipecal.edu.mx/Biblioteca/ Documento1.html Visitado 10 de Septiembre de 2010.

Gimeno Sacristán, J.F. (1982). La pedagogía por objetivos. Obsesión por la eficacia. Madrid. Morata.

Gimeno Sacristán, J. (2008). "Diez tesis sobre la aparente utilidad de las competencias en Educación". En Educar por competencias: ¿Qué hay de nuevo? Morata, Madrid España.

González, J. \& Wagenaar, R. (2003). (Eds.) Tuning Educational Structures in Europe. Informe Final- Fase I Bilbao: Universidad de Deusto.

González, J. \& Wagenaar, R. (2005). (Eds.) Tuning Educational Structures in Europe. Phase-II Bilbao, Universidad de Deusto.

Grundy, Shirley (1987). Curriculum: product or praxis. London. The Falmer Press.

Hartmann, E. (2008). Bologna goes global: a new imperialism in the making, Globalisation, Societies and Education, 6 (3), pp. 207-20.

Jones, Lynn \& Moore, Rob (2008). "La apropiación del significado de competencia: el movimiento de la competencia, la Nueva Derecha y el proyecto de 'cambio cultural"'. Profesorado. Revista de currículum y formación del profesorado: 12, 3 (http://www.ugr.es/local/recfpro/rev123ART7. pdf) (consultado el 27/06/10).

Nóvoa, António y de Johng-Lambert, William (2003). Educating Europe: an analysis of EU educational policies, en Phillips, David y Ertl, Hubert (2003). Implementing European Union education and training policy. A comparative study of issues in four member states. Dordrecht, The Netherlands. Kluwe Academic Publish.: 41-72.

Pérez Gómez, Ángel Ignacio (2007). La naturaleza de las competencias básicas y sus implicaciones pedagógicas. Consejería de Educación de Cantabria. Santander (http://www.redes-cepalcala. org/inspector/DOCUMENTOS\%20Y\%20LIBROS/COMPETENCIAS/NATURALEZA\%20 DE\%20LAS\%20COMPETENCIAS\%20BASICAS.pdf.) (visitado 15/09/10)

Pérez Gómez, Ángel Ignacio (2009). ¿Competencias o pensamiento práctico? La construcción de los significados de representación y de acción, en Gimeno Sacristán, José (2009) Educar por competencias, ¿qué hay de nuevo? Madrid. Morata: 59-102.

Power, Michael (1999). The audit society. Rituals of verification. Oxford. Oxford University Press.

Robertson, Susan L. (2006). "The politics of constructing (a competitive) Europe(an) through internationalising higher education: strategy, structures, subjects". Perspectives in Education, 24 (4), pp. 29-44.

Robertson, Susan L. (2008). The Bologna process goes global: a model, market mobility, brain power or state buiding strategy? Ponencia invitada a la conferencia anual ANPED. Octubre 2008. Brasil. Paper. 
Rubilar Solís, Luis (2009). La impertinencia de las competencias en educación y para la formación docente en Chile. www.umce.cl/luruso/impertinencia_competencias_chile.doc (consultado 21 de Septiembre de 2010).

Rychen, Dominique S. y Hersh Salganik, Laura (2003). Key competencies for a succesful life and a well-functioning society. Cambridge Hogrefer \& Huber.

Robertson, S. \& Keeling, R. (2008). Stirring the Lions: Strategy and Tactics in Global Higher Education. Globalisation, Societies and Education, v. 6, n. 3 p. 221-240.

Sennett, Richard (2006). La cultura del nuevo capitalismo. Editorial Anagrama, Barcelona España.

Stenhouse, Lawrence (1978). Investigación y desarrollo del currículo. Madrid. Morata.

Schön, Donald (1983). The reflective practitioner. How professionals think in action. London. Temple Smith.

Schön, Donald (1987). La formación de profesionales reflexivos. Hacia un nuevo diseño de la enseñanza y el aprendizaje en las profesiones. Barcelona. Paidós. 1992.

Stake, Robert (2006). Evaluación Comprensiva y evaluación basada en estándares. Editorial Graó, Barcelona España.

Stobart, Gordon (2010). Tiempos de pruebas: Los usos y abusos de la evaluación. Editorial Morata Madrid, España.

Tomasello, M.K. (2007). Los orígenes culturales de la cognición humana. B.A. Amorrortu.

Torres Santomé, Jurjo (2008). Obviando el debate sobre la cultura en el sistema educativo: cómo ser competentes sin conocimientos, en Gimeno Sacristán, J. (Comp.) (2008). Educar por competencias ¿qué hay de nuevo? Madrid. Morata: 143-175.

Toulmin, Stephen (2003). Regreso a la razón. El debate entre la racionalidad y la experiencia y la práctica personales en el mundo contemporáneo. Barcelona. Península.

UNESCO (1996). La educación encierra un tesoro. París Ediciones UNESCO.

Wheelahan, L. (2007). How competency-based training locks the working class out of powerful knowledge: a modified Bernsteinian analysis, British Journal of Sociology of Education. Volume 28, Issue 5 September 2007, pages 637-651.

Young, Michael F.D. (2008a). Bringing knowledge back in. From social constructivism to social realism in the sociology of education. Oxon. Routledge.

Young, Michael F.D. (2008b). From Constructivism to Realism in the Sociology of the Curriculum. Review of Research in Education, 32,1-28. 
